\title{
THE STRUCTURE OF CODIMENSION ONE DISTAL FLOWS WITH NON-TRIVIAL ISOTROPY
}

\author{
DENNIS F. DE RIGGI AND NELSON G. MARKLEY ${ }^{1}$
}

This paper grew out of our work on the following problem: Given a minimal action of $R^{n-1}(R=$ reals $)$ on a compact $n$-dimensional manifold $X$, what can you say about $X$. At present our results on the general problem are fragmentary, but with the additional assumption of distality we can completely determine the spaces and the structure of the actions, which is the subject of this paper. We proved in an earlier paper that distal minimal codimension one flows without any isotropy are equicontinuous; hence their structure is subsumed by the simple well known structure of minimal equicontinuous actions. This result plays a crucial role in our analysis when discrete non-trivial isotropy is present. In this situation we will show that $X$ must be the $n$-torus or belong to a special family of nilmanifolds.

In addition, these actions can all be realized as perturbations of canonical smooth distal codimension one flows. Alternatively, this says that regardless of the parameterization of the action we can always assume the orbits are the leaves of a foliation coming from commuting left invariant vector fields on a Lie group. This is analogous to the classical result that one can always assume the orbits of a minimal flow on the two-torus are translates of a dense one-parameter subgroup. We do not know whether or not a similar result holds when the action is not distal.

In section 1 we gather together the requisite basic facts about transformation groups, codimension one flows, imbeddings of discrete actions in flows, and cocycles. The second section is a pivotal one. In it we prove the existence of nice cocycle representations of the flows we are studying and establish a connection between the homotopy of these cocycles and the rank of the isotropic subgroup. This latter result is the key to determining which nilmanifolds can support minimal distal codimension one flows. The two natural classes-twist free and twisted-that arise from the representations obtained in section 2 are analyzed in sections 3 and 4 .

Some of the results in this paper were part of the first author's dissertation [1].

Received by the editors on July 7, 1977, and in revised form on February 2, 1978.

${ }^{1}$ The second author was supported by the National Science Foundation (MPS75-07078). 
1. Preliminaries. Let $Y$ be a metric space, let $T$ be a topological group, and let $(Y, T)$ be a transformation group. In other words, implicit in the notation $(Y, T)$ is the existence of a continuous map $(y, t) \rightarrow y t$ of $Y \times T$ into $Y$ such that $y e=y(e$ is the identity in $T)$ and $(y t) s=y(t+s)$ for all $y \in Y$ and all $t, s \in T$. The set $\{y t: t \in T\}$ will be denoted by $y T$. When $Y$ is compact we will speak of minimal and distal transformation groups; specifically, $(Y, T)$ is minimal if $\overline{y T}=Y$ for all $y \in Y$ and $(Y, T)$ is distal if $y t_{n} \rightarrow z$ and $x t_{n} \rightarrow z$ implies $y=x$.

Let $G$ be a compact topological group. By a bitransformation group we shall mean a triple $(G, Y, T)$ such that $(a)(Y, T)$ and $(G, Y)$ are transformation groups, (b) $(g y) t=g(y t)$ for all $g \in G, y \in Y, t \in T$, and (c) $g y=y$ for some $y \in Y$ implies $g$ is the identity.

We will denote the integers, reals, and complex numbers of modulus one by $Z, R$, and $K$ respectively. The only topological groups that will occur are $Z^{r}, R^{r}, K^{r}$ where $r$ is any positive integer. The group operation in $K^{r}$ will be denoted by $z \cdot w$ to avoid confusion with the action of a group on a space which is denoted by juxtaposition as in $x t$ or gx.

Let $X$ be a compact $n$-dimensional manifold without boundary. A transformation group $\left(X, R^{n-1}\right)$ will be called a codimension one flow. The subset $I_{x}=\left\{v \in R^{n-1}: x v=x\right\}$ is a closed subgroup of $R^{n-1}$. When $\left(X, R^{n-1}\right)$ is minimal, $I_{x}$ is independent of $x$ and will be denoted by $I$. If $\left(X, R^{n-1}\right)$ is minimal and $I$ is not discrete, then the codimension one aspect of $\left(X, R^{n-1}\right)$ is lost and the problem of determining the structure of $\left(X, R^{n-1}\right)$ is considerably altered. Consequently, we will always assume that minimal codimension one flows have discrete isotropy.

The objects of primary interest in this paper are the minimal distal codimension one flows $\left(X, R^{n-1}\right)$ with non-trivial isotropy, i.e., $I \neq\{0\}$. (If $I=\{0\}$, then $X$ is homeomorphic to $K^{n}$ and $\left(X, R^{n-1}\right)$ is equicontinuous [2, Corollary 4.6].) Since the subspace spanned by $I$ will play a special role, it will be convenient to represent $\left(X, R^{n-1}\right)$ as a bitransformation group. To do this choose a basis $\left\{e_{1}^{\prime}, \cdots, e_{p}{ }^{\prime}, \cdots, e_{n-1}^{\prime}\right\}$ for $R^{n-1}$ such that $\left\{e_{1}{ }^{\prime}, \cdots, e_{p}{ }^{\prime}\right\}$ are generators for $I$. Let $\exp t=e^{2 \pi i t} \in K$. Define $\left(K^{p}, X, R^{q}\right)$ where $q=n-1-p$ by $\left(x,\left(v_{1}\right.\right.$, $\left.\left.\cdots, v_{q}\right)\right) \rightarrow x\left(\sum_{i=1}^{q} v_{i} e_{p+i}^{\prime}\right)$ and $\left(\left(z_{1}, \cdots, z_{p}\right), x\right) \rightarrow x\left(\sum_{i=1}^{p} s_{i} e_{i}^{\prime}\right)$ where $\exp s_{i}=z_{i}, i=1, \cdots, p$. It is clear that this is a bitransformation group with the additional properties:

(a) $p+q=n-1=\operatorname{dim} X-1, q>0, p>0$.

(b) $t \in K^{p}, v \in R^{q}, x \in X$, and $t x v=x$

implies $t=(1, \cdots, 1)$ and $v=(0, \cdots, 0)$.

(c) $t x R^{q}: t \in K^{p}$ is a partition of $X$.

(d) $\left(X, R^{q}\right)$ is distal. 
Conversely, given a bitransformation group $\left(K^{p}, X, R^{q}\right)$ on a compact $n$ dimensional manifold as above, there is a natural minimal distal codimension one flow $\left(X, R^{n-1}\right)$ with non-trivial isotropy. Although most of the time we will use the bitransformation viewpoint, we will when necessary shift back and forth between these two viewpoints.

Let $Y$ be a compact metric space. There is a standard construction for imbedding $(Y, Z)$ in a continuous flow $\left(Y^{\prime}, R\right)$ of the form $Y \times[0,1] / \sim$. If you try to generalize this to imbedding $\left(Y, Z^{q}\right)$ in a flow $\left(Y^{\prime}, R^{q}\right)$ there is a more convenient way of making this construction, which we will now describe. Given $\left(Y, Z^{q}\right)$ define $\left(Y \times R^{q}, Z^{q}\right)$ by $(y, v) d \rightarrow(y d$, $v-d)$. This action of $Z^{q}$ on $Y \times R^{q}$ is clearly properly discontinuous and the quotient space $Y \times R^{q} / Z^{q}$ denoted by $\mathscr{I}\left(Y ; Z^{q}\right)$ is a compact metric space. The $R^{q}$ action on $Y \times R^{q}$ defined by $((y, w), v) \rightarrow(y, v+w)$ commutes with the $Z^{q}$ action and hence induces a flow $\left(\mathscr{I}\left(Y ; Z^{q}\right), R^{q}\right)$. Letting $\langle y, v\rangle$ denote the point of $\mathscr{I}\left(Y, Z^{q}\right)$ determined by $(y, v)$, it is easy to check that $\langle y, 0\rangle d=\langle y d, 0\rangle$. If $\phi$ is a homeomorphism of $Y$ onto itself such that $\phi(y d)=\phi(y) d$ for all $y \in Y$ and $d \in D$, then $\phi$ induces a homeomorphism $\hat{\phi}$ of $\mathscr{I}\left(Y ; Z^{q}\right)$ by $\hat{\phi}(\langle y, v\rangle)=\langle\phi(y), v\rangle$. Moreover, $\left(\phi^{\circ} \psi\right)=\hat{\phi}^{\circ} \hat{\psi}$ and $\left(\phi^{-1}\right)=\hat{\phi}^{-1}$. In particular, if $\left(G, Y, Z^{q}\right)$ is a bitransformation group then there is a natural action of $G$ on $\mathscr{I}\left(Y, Z^{q}\right)$ forming a bitransformation group $\left(G, \mathscr{I}\left(Y, Z^{q}\right), R^{q}\right)$. We also have the following proposition.

Proposition 1.1. If $H_{1}$ and $H_{2}$ are subgroups of $Z^{q}$ such that $Z^{q}=H_{1} \oplus H_{2}$, then $\mathscr{I}\left(Y ; Z^{q}\right)$ is homeomorphic to $\mathscr{I}\left(\mathscr{I}\left(Y ; H_{1}\right) ; \hat{H}_{1}\right)$. Moreover, if $\left(G, Y, Z^{q}\right)$ is a bitransformation group, then there is a $G$ equivariant homeomorphism with respect to natural $G$ actions on these spaces.

Proof. Use the map $\left\langle y, v_{1}+v_{2}\right\rangle \rightarrow\left\langle\left\langle y, v_{1}\right\rangle, v_{2}\right\rangle$ where $v_{i}$ is in the span of $H_{i}$.

If $\left\{\psi_{1}, \cdots, \psi_{q}\right\}$ are commuting homeomorphisms of $Y$ which generate $\left(Y, Z^{q}\right)$, then we will sometimes write $\mathscr{I}\left(Y ; \psi_{1}, \cdots, \psi_{q}\right)$ instead of $\mathscr{I}\left(Y ; Z^{q}\right)$.

It is well known that if $\phi$ and $\psi$ are isotopic homeomorphisms of $Y$, then $\mathscr{I}(Y ; \psi)$ and $\mathscr{I}(Y ; \phi)$ are homeomorphic. In fact, a homeomorphism is given by

$$
\langle y, t\rangle_{\phi} \rightarrow\langle h(y, t), t\rangle_{\psi}, \quad 0 \leqq t<1,
$$

where $h: Y \times[0,1] \rightarrow Y$ is an isotopy with $h(y, 0)=\psi(y)$ and $h(y, 1)=$ $\phi(y)$. It follows that if $\psi$ is isotopic to the identity, then $\mathscr{I}(Y, \psi)$ is homeomorphic to $Y \times K$. 
Let $(Y, T)$ be a transformation group with $Y$ compact. A cocycle of $(Y, T)$ into a compact group $G$ is a continuous function $\eta: Y \times T \rightarrow G$ such that

$$
\eta(y, s+t)=\eta(y s, t) \eta(y, s)
$$

for all $y \in Y$ and $s, t \in T$. For our purposes we want to assume that both $T$ and $G$ are abelian. All the cocycles of $(Y, T)$ into $G$ form a group under multiplication in $G$. A constant cocycle is by definition a continuous homomorphism of $T$ into $G$. Two cocycles $\eta$ and $\sigma$ are cohomologous \{weakly cohomologous\} if there exists a continuous function $h: Y \rightarrow G$ and an automorphism $\phi$ of $(Y, T)\}$ such that

$$
\begin{aligned}
\eta(y, t) \sigma(y, t)^{-1} & =h(y t) h(t)^{-1} \\
\left\{\eta(y, t) \sigma(\phi(y), t)^{-1}\right. & \left.=h(y t) h(y)^{-1}\right\}
\end{aligned}
$$

for all $y \in Y$ and $t \in T$.

Let $\left(G, Y^{\prime}, T\right)$ be a bitransformation group, let $Y=Y^{\prime} / G$, let $(Y, T)$ be the transformation group induced by $\left(Y^{\prime}, T\right)$, and let $\eta$ be a cocycle of $(Y, T)$ into $G$. Then we can define a new bitransformation group $\left(G Y^{\prime}, T_{\eta}\right)$ by $(y, t) \rightarrow \eta(p(y), t) y t$ where $p$ is the canonical map of $Y^{\prime}$ onto $Y$, and $\left(G, Y^{\prime}, T_{\eta}\right)$ is called the perturbation of $\left(G, Y^{\prime}, T\right)$ by the cocycle $\eta$. In particular, this makes sense when we are given $(Y, T)$ and we consider $(G, G \times Y, T)$ with the obvious actions. We can also speak of a perturbation of a minimal distal codimension one flow $\left(X, R^{n-1}\right)$ with non-trivial isotropy. This means that we consider an associated bitransformation group $\left(K^{p}, X, R^{q}\right)$, let $\eta$ be a cocycle of $\left(X / K^{p}, R^{q}\right)$ into $K^{p}$, form $\left(K^{p}, X, R_{\eta}^{q}\right)$, and from this obtain a comdimension one flow $\left(X, R_{\eta}{ }^{n-1}\right)$. It is easy to check that $\left(X, R_{\eta}{ }^{n-1}\right)$ will also be minimal distal. In fact, $\left(X, R^{n-1}\right)$ and $\left(X, R_{\eta}{ }^{n-1}\right)$ will have the same orbits.

Proposition 1.2. Let $(G, Y, T)$ be a bitransformation group. If $(y, t) \rightarrow y^{*} t$ is another action on $Y$ such that $g\left(y^{*} t\right)=(g y)^{*} t$ and $G(y t)$ $=G\left(y^{*} t\right)$ for all $y \in Y$ and $t \in T$, then there exists a cocycle $\eta$ of $(Y / G, T)$ into $G$ such that $y^{*} t=\eta(G y, t) y t$.

Proof. Given $y$ and $t$ there exists a unique $k(y, t) \in G$ such that $y^{*} t=k(y, t) y t$. Since $g\left(y^{*} t\right)=(g y)^{*} t, g k(y, t) y t=k(g y, t) g y t$ and hence $k(y, t)=k(g y, t)$ for all $g \in G$. Therefore there exists a function $\eta: Y / G \times T \rightarrow G$ such that $y^{*} t=\eta(G y, t) y t$. It is straightforward to check that $\eta$ is a cocycle of $(Y / G, T)$.

Proposition 1.3. Given $(Y, T)$ and two cocycles $\eta$ and of of $(Y, T)$ into $G$, then the bitransformation groups $\left(G, G \times Y, T_{\eta}\right)$ and $\left(G, G \times Y, T_{\sigma}\right)$ 
obtained from the canonical $(G, G \times Y, T)$ are isomorphic if and only if $\eta$ and $\sigma$ are weakly cohomologous.

Proof. Let $\psi$ be an isomorphism of $\left(G, G \times Y, T_{\eta}\right)$ onto $(G, G \times Y$, $\left.T_{\sigma}\right)$. Then $\psi$ induces an automorphism $\psi_{0}$ of $(Y, T)$ such that $\psi(g, y)=$ $\left(h(g, y) g, \psi_{0}(y)\right)$. Since $\psi(g, y)=g \psi(e, y)$, one easily checks that $h(g y)=h(e, y)$ and that $h$ is the required function relating $\eta$ and $\sigma$. For the converse one simply uses the above formula to define $\psi$.

2. Internal Structure. Let $\left(K^{p}, X, R^{q}\right)$ be a bitransformation group associated with a minimal distal codimension one flow with non-trival isotropy of rank $p$. In this section we will prove the existence of a torus $M$ in $X$ and a discrete subgroup $D$ of $R^{q}$ of rank $q$ such that $M$ is invariant under both $K^{p}$ and $D$. The rest of the paper is based on the structure of $\left(K^{p}, M, D\right)$ which will also be exposed in this section.

Let $X / K^{p}$ denote the quotient space obtained by identifying the $K^{p}$ orbits.

Theorem 2.1. The space $X / K^{p}$ is homeomorphic to $K^{q+1}$ and $\left(X / K^{p}\right.$, $\left.R^{q}\right)$ is equicontinuous.

Proof. Since $\left(X / K^{p}, R^{q}\right)$ is distal with trivial isotropy,, it suffices by $\left[2\right.$, Corollary 4.6] to show that $X / K^{p}$ is a $(q+1)$-dimensional manifold. This is an easy consequence of the following facts: the canonical map from $X$ to $X / K^{p}$ is open and there exist local sections for $\left(X, R^{n-1}\right)$ homeomorphic to $[0,1]$ (See [2, Lemma 4.1]).

It follows from this theorem and [2, Proposition 1.3] that there exists a discrete syndetic subgroup $D$ of $R^{q}$ such that $X / K^{p}$ is not minimal un$\operatorname{der} D$ and $y t \in \overline{y D}$ implies $t \in D$ for any $y \in X / K^{p}$. Note that the rank of $D$ must be $q$. Moreover, $D$ can be chosen so that $\overline{y D}$ is homeomorphic to $K$ (see the proof of Theorem 4.2 in [2]). Pick $y_{o} \in X / K^{p}$ and set $C=\overline{y_{0} D}$. There exists a lift $\tilde{C}$ of $C$ to $X$ because $p: X \rightarrow X / K^{p}$ is a fibration with an arcwise connected fiber. Set $M=K^{p} \tilde{C}=$ $\left\{w x: w \in K^{p}\right.$ and $\left.x \in \tilde{C}\right\}$. Clearly $M=p^{-1}(C)$. It is easy to see that $(w, x) \rightarrow w x$ is a homeomorphism of $K^{p} \times \tilde{C}$ onto $M$. Thus $M$ is homeomorphic to $K^{p+1}, M$ is invariant under $K^{p}$, and $M$ is invariant under $D$. By choosing our basis $\left\{e_{1}{ }^{\prime}, \cdots, e_{n-1}^{\prime}\right\}$ with a little more care we can assume without loss of generality that $D=Z^{q}$. We can now form the bitransformation group $\left(K^{p}, \mathscr{I}\left(M ; Z^{q}\right), R^{q}\right)$.

Proposition 2.2. The bitransformation groups $\left(K^{p}, X, R^{q}\right)$ and $\left(K^{p}\right.$, $\left.\mathscr{I}\left(M, Z^{q}\right) R^{q}\right)$ are isomorphic.

Proof. The map $\Lambda: M \times R^{q} \rightarrow X$ given by $\Lambda(m, v)=m v$ is a homomorphism of $\left(K^{p}, M \times R^{q}, R^{q}\right)$ onto $\left(K^{p}, X, R^{q}\right)$. Suppose $\Lambda(m, v)=$ 
$\Lambda\left(m^{\prime}, v^{\prime}\right)$, i.e., $m v=m^{\prime} v^{\prime}$. Then $m=m^{\prime}\left(v^{\prime}-v\right)$ implies $v^{\prime}-v=$ $d \in Z^{q}$ because $p(m)=p\left(m^{\prime}\right)\left(v^{\prime}-v\right)$ with $p(m)$ and $p\left(m^{\prime}\right)$ in $C$. Thus $(m, v) d=\left(m^{\prime}, v^{\prime}\right)$. Conversely $\Lambda(m, v)=\Lambda(m d, v-d)$ for all $d \in R^{q}$.

Now we study $\left(K^{p}, M, Z^{q}\right)$. By Lemma 4.4 in [2] there exists a homeomorphism $\mu: C \rightarrow K$ and a homomorphism (group) $\theta: Z^{q} \rightarrow K$ such that $\mu(y d)=\mu(y) \cdot \theta(d)$. If $\theta(d)=1$, then $\mu(y d)=\mu(y)$ implies $y d=y$ and $d \in D \cap I=\{0\}$. Define a homeomorphism $h: M \rightarrow K^{p+1}$ by $h(m)=\left(z_{0}, \cdots, z_{p}\right)$ if and only if $\left(z_{1}, \cdots, z_{p}\right) m \in \tilde{C}$ and $\mu(p(m))=z_{0}$. This forces $h$ to be an isomorphism of $\left(K^{p}, M\right)$ onto $\left(K^{p}, K^{p+1}\right)$ where the latter action is coordinate-wise multiplication on the last $p$-coordinates. Letting $\phi_{d}$ be the conjugate by $h$ of the action of $d$ on $M$, we see that

$$
\begin{aligned}
\phi_{d}\left(z_{0},\right. & \left.z_{1}, \cdots, z_{p}\right)=h\left(h^{-1}\left(z_{0}, \cdots, z_{p}\right) d\right) \\
& =\left(z_{1}, \cdots, z_{p}\right) h\left(h^{-1}\left(z_{0}, 1, \cdots, 1\right) d\right) \\
& =\left(z_{1}, \cdots, z_{p}\right)\left(z_{0} \cdot \theta(d), f_{1}\left(z_{0}, d\right), \cdots, f_{p}\left(z_{0}, d\right)\right) \\
& =\left(z_{0} \cdot \theta(d), z_{1} \cdot f_{1}\left(z_{0}, d\right), \cdots, z_{p} \cdot f_{p}\left(z_{0}, d\right)\right)
\end{aligned}
$$

where each $f_{i}: K \times Z^{q} \rightarrow K$ is continuous. Thus $h$ allows us to represent $\left(K^{p}, M, Z^{q}\right)$ by complex multiplication and a skew product type construction. It is easy to check that $f=\left(f_{1}, \cdots, f_{p}\right)$ is a cocycle of $\left(K, Z^{q}\right)$ where $(z, d) \rightarrow z \cdot \theta(d)$. This establishes the following theorem:

TheOREM 2.3. Let $\left(X, R^{n-1}\right)$ be a minimal distal codimension one flow for which the rank of I equals $p>0$. Then there exists a basis $\left\{e_{1}^{\prime}\right.$, $\left.\cdots, e_{n-1}^{\prime}\right\}$ or $\mathrm{R}^{n-1}$ such that the associated bitransformation group $\left(\mathrm{K}^{p}\right.$, $\left.X, R^{q}\right)$ is isomorphic to a bitransformation group $\left(K^{p}, \mathscr{I}\left(K^{p+1} ; Z^{q}\right), R^{q}\right)$ for which the action of $Z^{q}$ on $K^{p+1}$ is of the form $\left(\left(z_{0}, \cdots\right.\right.$, $\left.\left.z_{p}\right), d\right) \rightarrow\left(z_{0} \cdot \theta(d), f_{1}\left(z_{0}, d\right) \cdot z_{1}, \cdots, f_{p}\left(z_{0}, d\right) \cdot z_{p}\right)$, where $\theta: Z^{q} \rightarrow K$ is an isomorphism and $f: K \times Z^{q} \rightarrow K^{p}$ is a cocycle of $\left(K, Z^{q}\right)$ given by $(z, d) \rightarrow z \cdot \theta(d)$ and the action of $K^{p}$ on $K^{p+1}$ is given by $\left(\left(w_{1}, \cdots, w_{p}\right)\right.$, $\left.\left(z_{0}, \cdots, z_{p}\right)\right) \rightarrow\left(z_{0}, w_{1} \cdot z_{1}, \cdots, w_{p} \cdot z_{p}\right)$.

A representation of $\left(X, R^{n-1}\right)$ as a bitransformation of the form described in he above theorem will be called a cocycle representation. We will say $\left(\mathrm{X}, \mathrm{R}^{n-1}\right)$ is twist free if it has a cocycle representation given by a cocycle $f=\left(f_{1}, \cdots, f_{p}\right)$ such that the degree of $z \rightarrow f_{i}(z, d)$ is zero for all $d$ in $Z^{q}$ and $1 \leqq i \leqq p$. If this is not the case we will say $\left(X, R^{n-1}\right)$ is twisted.

The integer $p$ (and hence $q$ ) is an invariant of the original flow. The monomorphism $\theta$ is a generalized rotation number, but it is not an in- 
variant because it depends upon our choice of basis for $R^{n-1}$ and of the discrete subgroup $D$. However, for each $p, 0<p<n-1$, and each monomorphism $\theta: Z^{q} \rightarrow K$ we can construct a class of minimal distal codimension one flows on $n$-dimensional manifolds by using the above procedure. We have already shown that every minimal distal codimension one flow with non-trivial isotropy belongs to such a class. In the remainder of this section we will begin our study of these classes by examining the underlying bitransformation $\left(K^{p}, K^{p+1}, Z^{q}\right)$.

Let $p$ be a fixed integer between 0 and $n-1$ and let $\theta: Z^{q} \rightarrow K$ be a fixed monomorphism where $q=n-1-p$. Define $\left(K, Z^{q}\right)$ by $(z, d) \rightarrow z \cdot \theta(d)$. For each cocycle $f: K \times Z^{q} \rightarrow K^{p}$ of $\left(K, Z^{q}\right)$ define the bitransformation group $\left(K^{p}, K^{p+1}, Z_{f}^{q}\right)$ by $\left(\left(w_{1}, \cdots, w_{p}\right),\left(z_{0}, \cdots, z_{p}\right)\right) \rightarrow$ $\left(z_{0}, w_{1} \cdot z_{1}, \cdots, w_{p} \cdot z_{p}\right) \quad$ and $\quad\left(\left(z_{0}, \cdots, z_{p}\right), d\right) \rightarrow\left(z_{0} \cdot \theta(d), \quad z_{1} \cdot f_{1}\left(z_{0}, d\right)\right.$, $\left.\cdots, z_{p} \cdot f_{p}\left(z_{0}, d\right)\right)$.

TheOREM 2.4. Let $f$ be a cocycle of $\left(K, Z^{q}\right)$ into $K^{p}$. If for some $d \in Z^{q}$ and $i, 1 \leqq i \leqq p$ the degree of $z \rightarrow f_{i}(z, d)$ is non-zero where $f=\left(f_{1}, \cdots, f_{p}\right)$, then $q=1$.

Proof. Suppose $\operatorname{deg} f_{i}(, d)=n \neq 0$. Choose $d^{\prime}$ so that $\theta(d)=\alpha$ and $\theta\left(d^{\prime}\right)=\beta$ are rationally independent which is possible if $q>1$. Let $m=\operatorname{deg} f_{i}\left(, d^{\prime}\right)$, let $F_{i}(x, d)$ and $F_{i}\left(x, d^{\prime}\right)$ be lifts of $f_{i}(z, d)$ and $f_{i}\left(z d^{\prime}\right)$, and set $F_{i}{ }^{\prime}(x, d)=F_{i}(x, d)-n x$ and $F_{i}{ }^{\prime}\left(x, d^{\prime}\right)=F_{i}\left(x, d^{\prime}\right)-m x$. Since $f$ is a cocycle of $\left(K, Z^{q}\right)$,

$$
f_{i}\left(z \cdot \alpha, d^{\prime}\right) \cdot f_{i}(z, d)=f_{i}(z \cdot \beta, d) \cdot f_{i}\left(z, d^{\prime}\right)
$$

and there exists an integer $N$ such that

$$
F_{i}\left(x+a, d^{\prime}\right)+F_{i}(x, d)=F_{i}(x+b, d)+F_{i}\left(x, d^{\prime}\right)+N,
$$

where $\exp a=\alpha$ and $\exp b=\beta$. From this we get

$$
\begin{aligned}
F_{i}{ }^{\prime}(x & \left.+a, d^{\prime}\right)+F_{i}{ }^{\prime}(x, d)+m a \\
& =F_{i}{ }^{\prime}(x+b, d)+F_{i}{ }^{\prime}\left(x, d^{\prime}\right)+n b+N .
\end{aligned}
$$

Since $F_{i}{ }^{\prime}(x+1, d)=F_{i}{ }^{\prime}(x, d)$ and $F_{i}{ }^{\prime}\left(x+1, d^{\prime}\right)=F_{i}{ }^{\prime}(x, d)$ for all $x$, upon taking the integral from 0 to 1 of the above we have

$$
m a=n b+N
$$

This gives us the contradiction $\alpha^{m}=\beta^{n}, n \neq 0$.

CoRollary 2.5. If $\left(X, R^{n-1}\right)$ is a twisted minimal distal codimension one flow, then the rank of its isotropy is $n-2$. 
Theorem 2.6. There exist cocycles of $\left(K, Z^{q}\right)$ into $K^{p}$ which are not cohomologous to a constant.

Proof. Since $\sigma=\left(\sigma_{1}, \cdots, \sigma_{p}\right)$ is a cocycle if and only if each $\sigma_{i}$ is a cocycle, it suffices to assume $p=1$. Also the proof for arbitrary $q$ will be clear from the proof for $q=2$.

Let $\alpha=\theta\left(d_{1}\right)$ and $\beta=\theta\left(d_{2}\right)$ where $d_{1}=(1,0)$ and $d_{2}=(0,1)$. It suffices to find two continuous functions $f, g: K \rightarrow K$ of degree zero such that

$$
f(z \cdot \beta) \cdot g(z)=g(z \cdot \alpha) \cdot f(z)
$$

because then we can set $\sigma\left(z, d_{1}\right)=f(z)$ and $\sigma\left(z, d_{2}\right)=g(z)$ and use the cocycle formula to define $\sigma(z, d)$ for arbitrary $d$ in $Z^{2}$. Let $F, G: K \rightarrow R$ be lifts of $f$ and $g$. Then

$$
F(z \cdot \beta)+G(z)=G(z \cdot \alpha)+F(z)+N
$$

for some $N \in Z$, and by taking the Haar integral of both sides we see that $N=0$. Letting $a_{n}$ and $b_{n}$ be the Fourier coefficients of $F$ and $G$ we have

$$
\begin{aligned}
& b_{n}=\frac{1-\beta^{n}}{1-\alpha^{n}} a_{n}, \quad n \neq 0 \\
& a_{n}=\bar{a}_{-n}, \\
& b_{n}=\bar{b}_{-n} .
\end{aligned}
$$

We must also ensure that for any constant $\xi \in K$,

$$
f(z) / \xi=h(z \cdot \alpha) / h(z)
$$

has no continuous solution $h: K \rightarrow K$. Assume for the moment such an $h$ exists. Then there exists $H: K \rightarrow R$ which is continuous except at $z=1$ and satisfies $\exp (H(z))=h(z)$, and we have

$$
F(z)=H(z \cdot \alpha)-H(z)+N^{\prime}
$$

for some $N^{\prime}$. Clearly $N^{\prime}=a_{0}$. Letting $c_{n}$ denote the $n^{\text {th }}$ Fourier coefficient of $H$, we have

$$
c_{n}=\frac{a_{n}}{\alpha^{n}-1}, \quad n \neq 0 .
$$

Thus it suffices to find a sequence of positive integers $\left\{n_{j}\right\}$ such that

$$
\sum_{j=1}^{\infty}\left|\alpha^{n_{j}}-1\right|<\infty
$$


and

$$
\left\{\left|\frac{1-\beta^{n_{j}}}{1-\alpha^{n_{j}}}\right|\right\}_{j=1}^{\infty} \text { is bounded, }
$$

because we can set

$$
a_{n}= \begin{cases}\alpha^{n}-1 & \text { if } n= \pm n_{j} \\ 0 & \text { otherwise }\end{cases}
$$

which would determine both $F$ and $G$ and which would guarantee that $a_{n} / \alpha^{n}-1$ cannot be the Fourier coefficients of an $L^{1}$ function. For each positive $j$ in $Z$ we can choose a positive $n_{j}$ in $Z$ such that $\left(\alpha^{n_{i}}, \beta^{n_{j}}\right)$ is in the open set

$$
\left\{(z, w) \in K^{2}: \frac{1}{2^{k+1}}<|z-1|<|w-1|<\frac{1}{2^{k}}\right\}
$$

because $\alpha$ and $\beta$ are rationally independent. It follows immediately that such a sequence satisfies (a) and (b) to complete the proof.

Theorem 2.7. Let $\sigma: K \times Z^{q} \rightarrow K^{p}$ be a cocycle of $\left(K, Z^{q}\right)$. Then $\left(K^{p+1}, Z^{q}\right)$ is equicontinuous if and only if $\sigma$ is cohomologous to a constant.

Proof. The sufficiency is obvious and the necessity follows by applying a recent result of Ellis [3, Theorem 3.21] to a minimal subset of $\left(K^{p+1}, Z^{q}\right)$.

3. The Twist Free Case. This section is devoted to determining the underlying space and the structure of the flow when $\left(X, R^{n-1}\right)$ is a twist free minimal distal codimension one flow with non-trivial isotropy. When $p=\operatorname{rank} I=n-2$ it is obvious in this case that $X$ is an $n$ torus. Moreover, since $\mathscr{I}(Y ; \phi, \psi)$ is homeomorphic to $\mathscr{I}(\mathscr{I}(Y, \phi), \hat{\psi})$ (Proposition 1.1), we could get the same result for any $p$ provided we know $\hat{\psi}$ is isotopic to the identity. In the beginning of this section we present a technical theorem which both resolves this difficulty and allows us to show that these flows are perturbations of equicontinuous ones.

Let $S(m, N)$ be the homeomorphisms of $K^{m+N+1}$ which are of the form

$$
\begin{aligned}
& \phi\left(z_{0}, z_{1}, \cdots, z_{m}, w_{1}, \cdots, w_{N}\right)= \\
& =\left(\alpha \cdot z, f_{1}\left(z_{0}, w_{1}, \cdots, w_{N}\right) \cdot z_{1}, \cdots,\right. \\
& \left.f_{m}\left(z_{0}, w_{1}, \cdots, w_{N}\right) \cdot z_{m}, w_{1}, \cdots, w_{N}\right),
\end{aligned}
$$


where $\alpha \in K$ and each $f_{i}$ is continuous. For brevity, $\phi$ will be written $\left(\alpha, f_{1}, \cdots f_{m}\right)$, and $S^{\circ}(m, N)$ will denote the subset of $S(m, N)$ for which every $f_{i}$ is null homotopic. It is easy to check that $S(m, N)$ is a group under composition of maps and $S^{\circ}(m, N)$ is a subgroup.

Proposition 3.1. Let $\phi=\left(\alpha, f_{1}, \cdots, f_{m}\right)$ and $\psi=\left(\beta, g_{1}, \cdots, g_{m}\right)$ be the elements of $S(m, N)\left\{S^{\circ}(m, N)\right\}$. If $f_{i}$ is homotopic to $g_{i}$ for $i=1$, $\cdots, n$, then $\phi$ is isotopic to $\psi$ in $S(m, N)\left\{S^{\circ}(m, N)\right\}$.

Proof. Let $h_{j}\left(z_{0}, w_{1}, \cdots, w_{N}, t\right)$ be a homotopy between $f_{j}$ and $g_{j}$. Choose $a, b \in R$ such that $\exp (a)=\alpha$ and $\exp (b)=\beta$ and define $\alpha^{t}=\exp (t a)$ and $\beta^{t}=\exp (t b)$. Set $H\left(z_{0}, z_{1}, \cdots, z_{m}, w_{1}, \cdots, w_{N}, t\right)=$ $\left(\alpha^{1-t} \cdot \beta^{t} \cdot z_{0}, h_{1}\left(z_{0}, w_{1}, \cdots, w_{N}, t\right) \cdot z_{1}, \cdots, h_{m}\left(z_{0}, w_{1}, \cdots, w_{n}, t\right) \cdot z_{m}\right.$, $\left.w_{1}, \cdots, w_{N}\right)$.

Corollary 3.2. Let $\phi=\left(\alpha, f_{1}, \cdots, f_{m}\right)$ and $\psi=\left(\beta, g_{1}, \cdots, g_{m}\right)$ be elements of $\mathrm{S}(m, N)$. If $f_{i}$ is homotopic to $\mathrm{g}_{i}$ for $i=1, \cdots, m$, then $\mathscr{I}\left(K^{m+N+1} ; \phi\right)$ is homeomorphic to $\mathscr{I}\left(K^{m+N+1} ; \psi\right)$. In particular, if $\phi \in S^{\circ}(m, n)$, then $\mathscr{I}\left(K^{m+N+1} ; \phi\right)$ is homeomorphic to $K^{m+N+2}$.

Suppose $\phi \in S^{\circ}(m, N)$. We will need the explicit homeomorphism of $\mathscr{I}\left(K^{m+N+1} ; \phi\right)$ onto $K^{m+N+2}$ given by

$$
\zeta\left(\langle y, t\rangle_{\phi}\right)=(H(y, t), \exp t)
$$

where $H$ is the isotopy constructed in the proof of Proposition 3.1 such that $H(y, 0)$ is the identity and where each $h_{j}$ is of the form $h_{j}\left(z_{0}, w_{1}\right.$, $\left.\cdots, w_{N}, t\right)=\exp \left(t \tilde{f}_{j}\left(z_{0}, w_{1}, \cdots, w_{N}\right)\right)$. We also have

$$
\begin{gathered}
\zeta^{-1}\left(z_{0}, z_{1}, \cdots, z_{m}, w_{1}, \cdots, w_{N}, \exp (t)\right) \\
=\left\langle z_{0} \alpha^{-t}, \exp \left(-t \tilde{f}_{j}\left(\alpha^{-t} z_{0}, w_{1}, \cdots, w_{N}\right)\right) \cdot z_{1}, \cdots, w_{1}, \cdots, w_{N}, t\right\rangle_{\phi} .
\end{gathered}
$$

Theorem 3.3. Let $\phi=\left(\alpha, f_{1}, \cdots, f_{m}\right)$ and $\psi=\left(\beta, g_{1}, \cdots, g_{m}\right)$ be elements of $S^{\circ}(m, N)$. If $\phi^{\circ} \psi=\psi \circ \phi$, then $\bar{\psi}=\zeta^{\circ} \hat{\psi}^{\circ} \zeta^{-1}$ is an element of $S^{\circ}(m, N+1)$.

Proof. From the commutativity of $\phi$ and $\psi$ we see that

$$
\frac{f_{i}\left(z_{0}, w_{1}, \cdots, w_{N}\right)}{f_{i}\left(\beta \cdot z_{0}, w_{1}, \cdots, w_{N}\right)}=\frac{g_{i}\left(z_{0}, w_{1}, \cdots, w_{N}\right)}{g_{i}\left(\alpha \cdot z_{0}, w_{1}, \cdots, w_{N}\right)}
$$

and hence

$$
\begin{gathered}
\tilde{f}_{i}\left(z_{0}, w_{1}, \cdots, w_{N}\right)-\tilde{f}_{i}\left(\beta \cdot z_{0}, w_{1}, \cdots, w_{N}\right)= \\
\tilde{g}_{i}\left(z_{0}, w_{1}, \cdots, w_{N}\right)-\tilde{g}_{i}\left(\alpha \cdot z_{0}, w_{1}, \cdots, w_{N}\right)+K
\end{gathered}
$$


for some $k \in Z$. By taking the Haar integral we see that $k=0$. Define $\tilde{\omega}_{i}: K^{N+1} \times[0,1] \rightarrow R$ by

$$
\begin{aligned}
\tilde{\omega}_{i}\left(z_{0}, w_{1}, \cdots, w_{N}, t\right)=\tilde{g}_{i}\left(z_{0} \cdot \alpha^{-t}, w_{1}, \cdots, w_{N}\right) \\
\quad+t\left[\tilde{f}_{i}\left(z_{0} \cdot \alpha^{-t} \cdot \beta, w_{1}, \cdots, w_{N}\right)-\tilde{f}_{i}\left(z_{0} \cdot \alpha^{-t}, w_{1}, \cdots, w_{N}\right)\right] \\
=g_{i}\left(z_{0} \cdot \alpha^{-t}, w_{1}, \cdots, w_{N}\right) \\
\quad+t\left[g_{i}\left(z_{0} \cdot \alpha^{1-t}, w_{1}, \cdots, w_{N}\right)-\tilde{g}_{i}\left(z_{0} \alpha^{-t}, w_{1}, \cdots, w_{N}\right)\right] .
\end{aligned}
$$

The last equality holds because $k=0$. Note that $\tilde{\omega}_{i}\left(z_{0}, w_{1}, \cdots, w_{N}, 0\right)$ $=\tilde{\omega}_{i}\left(z_{0}, w_{1}, \cdots, w_{N}, 1\right)=\tilde{g}_{i}\left(z_{0}, w_{1}, \cdots, w_{N}\right)$ and hence we can think of $\tilde{\omega}_{i}: K^{N+2} \rightarrow R$. Let $\omega_{i}=\exp \left(\tilde{\omega}_{i}\right)$ which is clearly null homotopic and which can be written

$$
\begin{aligned}
& \omega_{j}\left(z_{0}, w_{1}, \cdots, w_{N}, \exp (t)\right)= \\
& \frac{g_{j}\left(z \cdot \alpha^{-t}, w_{1}, \cdots, w_{N}\right) \cdot \exp \left(t \tilde{f}_{j}\left(z_{0} \cdot \beta \cdot \alpha^{-t}, w_{1}, \cdots, w_{N}\right)\right)}{\exp \left(t \tilde{f}_{j}\left(z_{0} \cdot \alpha^{-t}, w_{1}, \cdots, w_{N}\right)\right)}
\end{aligned}
$$

Let $\theta=\left(\beta, \omega_{1}, \cdots, \omega_{m}\right) \in S^{\circ}(m, N+1)$. The proof is completed by the routine calculation that $\theta \circ \zeta=\zeta \circ \hat{\psi}$.

Corollary 3.4. If $\left(X, R^{n-1}\right)$ is a twist free minimal distal codimension one flow with non-trivial isotropy, then $X$ is homeomorphic to $K^{n}$.

Proof. This follows from Theorem 2.3, Proposition 1.1, and Corollary.

Theorem 3.5. Given any positive integers $p$ and $n$ such that $1 \leqq p \leqq n-2$ there exists a minimal distal but not equicontinuous codimension one flow on $K^{n}$ whose isotropy has rank $p$.

Proof. This follows from Theorems 2.6, 2.7, and 3.4.

We now turn to the dynamical structure of these flows. Let $p$ and $n$ be positive integers such that $1 \leqq p \leqq n-2$, and let $\theta: Z^{q} \rightarrow K$ be a monomorphism where $q=n-1-p$. The homomorphism of $R^{q}$ into $K^{n}$ given by $\left(v_{1}, \cdots, v_{q}\right) \rightarrow\left(\exp \left(\sum_{i=1}^{q} a_{i} v_{i}\right), 1, \cdots, 1, \exp v_{1}, \cdots\right.$, $\left.\exp v_{q}\right)$ where $\exp a_{i}=\theta\left(e_{i}\right)$ and $\left\{e_{1}, \cdots, e_{q}\right\}$ are the standard generators of $Z^{q}$ induces an equicontinuous flow $\left(K^{n}, R^{q}\right)$ by multiplication in $K^{n}$. In what follows $\left(K^{m}, K^{m+r+1}\right)$ will always denote the $K^{m}$ action on $K^{m+r+1}$ given by $\left(\left(w_{1}, \cdots, w_{m}\right),\left(z_{0}, \cdots, z_{m+r}\right)\right) \rightarrow\left(z_{0}, w_{1} \cdot z_{1}, \cdots\right.$, $\left.w_{m} z_{m}, z_{m+1}, \cdots, z_{m+r}\right)$. Hence $\left(K^{p}, K^{n}, R^{q}\right)$ is a bitransformation group associated with a minimal equicontinuous codimension one flow whose isotropy has rank $p$. Let $f: K \times Z^{q} \rightarrow K^{p}$ be a cocycle of $\left(K, Z^{q}\right)$ such 
that each $f(\cdot, d)$ has degree zero. We will show that $\left(K^{p}, \mathscr{I}\left(K^{p+1} ; Z_{f}^{q}\right)\right.$, $\left.R^{q}\right)$ can be realized as a perturbation of $\left(K^{p}, K^{n}, R^{q}\right)$.

Let $\phi_{i}=\left(\alpha_{i}, f_{i 1}, \cdots, f_{i m}\right) i=1, \cdots, q$ be commuting elements of $S^{\circ}(m, N)$, and let $\phi_{i}{ }^{\prime}=\left(\alpha_{i}\right) \in S^{\circ}(0, N)$. Define $\Pi^{\prime}: \mathscr{I}\left(K^{m+N+1} ; \phi_{1}, \cdots\right.$, $\left.\phi_{q}\right) \rightarrow \mathscr{I}\left(K^{N+1} ; \phi_{1}{ }^{\prime}, \cdots, \phi_{q}{ }^{\prime}\right)$ by $\Pi^{\prime}\left(\left\langle z_{0}, z_{1}, \cdots, z_{m}, w_{1}, \cdots, w_{N}, v\right\rangle\right)=$ $\left\langle z_{0}, w_{1}, \cdots, w_{N}, v\right\rangle$. It is immediate that $\Pi^{\prime}$ is a homomorphism of $\left(\mathscr{I}\left(K^{m+N+1} ; \phi_{1}, \cdots, \phi_{q}\right), R^{q}\right)$ onto $\left(\mathscr{I}\left(K^{N+1} ; \phi_{1}{ }^{\prime}, \cdots, \phi_{q}{ }^{\prime}\right), R^{q}\right)$ and identifies the latter with $\left(\mathscr{I}\left(K^{m+N+1} ; \phi_{1}, \cdots, \phi_{q}\right) / K^{m}, R^{q}\right)$. We can also define $\Psi:\left(\mathscr{I}\left(K^{N+1} ; \phi_{1}{ }^{\prime}, \cdots, \phi_{q}{ }^{\prime}\right), R^{q}\right) \rightarrow\left(K^{N+1+q}, R^{q}\right)$, where the latter action is defined by homomorphism $v \rightarrow\left(\exp \sum_{i=1}^{q} a_{i} v_{i}, 1, \cdots, 1, \exp v_{1}\right.$, $\left.\cdots, \exp v_{q}\right)$ with $\exp a_{i}=\alpha_{i}$, by

$$
\begin{gathered}
\Psi\left(\left\langle z_{0}, w_{1}, \cdots, w_{N}, v\right\rangle\right)= \\
\left(z_{0} \cdot \exp \left(\sum_{i=1}^{q} a_{i} v_{i}\right), w_{1}, \cdots, w_{N}, \exp v_{1}, \cdots, \exp v_{q}\right) .
\end{gathered}
$$

It is easy to see that $\psi$ is an isomorphism. Finally let $\Pi: K^{m+N+1} \rightarrow K^{N+1}$ be the projection $\Pi\left(z_{0}, z_{1}, \cdots, z_{m}, w_{1}, \cdots, w_{N}\right)=$ $\left(z_{0}, w_{1}, \cdots, w_{N}\right)$. In this context we have the following lemma:

LemMA 3.6. There exists an isomorphism $\Theta$ of $\left(K^{m}, \mathscr{I}\left(K^{m+N+1} ; \phi_{1}\right.\right.$, $\left.\cdots, \phi_{q}\right)$ onto $\left(K^{m}, K^{m+N+q+1}\right)$ such that $\Pi \circ \theta=\Psi \circ \Pi^{\prime}$.

Proof. We use induction on $q$ with $m$ and $N$ arbitrary. When $q=1$, the conclusion holds with $\theta=\zeta$ because of the multiplicative nature of $\zeta$. Assuming it holds for $q-1$ we will construct $\theta$. First there exists a canonical isomorphism $\Theta_{1}:\left(K^{m}, \mathscr{I}\left(K^{m+N+1} ; \phi_{1}, \cdots, \phi_{q}\right)\right)$ onto $\left(K^{m}\right.$, $\mathscr{I}\left(\mathscr{F}\left(K^{m+N+1} ; \phi_{1}\right) ; \hat{\phi}_{2}, \cdots, \hat{\phi}_{q}\right)$. From the $q=1$ case we get a canonical isomorphism $\Theta_{2}$ of the latter onto $\left(K^{m,} \mathscr{I}\left(K^{m+N+2} ; \bar{\phi}_{2}, \cdots, \bar{\phi}_{q}\right)\right)$. Finally Theorem 3.3 allows one to apply the induction hypothesis to $\left(K^{m}, \mathscr{I}\left(K^{m+N+2} ; \bar{\phi}_{2}, \cdots, \bar{\phi}_{q}\right)\right.$ to get an isomorphism $\theta_{3}$ of it onto $\left(K^{m}\right.$, $\left.K^{m+N+q+1}\right)$. Let $\theta=\theta_{3} \circ \theta_{2} \circ \theta_{1}$. It remains to show that $\Pi \circ \theta=$ $\Psi \circ \Pi^{\prime}$.

Each $\Theta$ induces a homeomorphism $\Psi_{i}$. They go from $\mathscr{T}\left(K^{N+1} ; \phi_{1}{ }^{\prime}\right.$, $\left.\cdots, \phi_{q}{ }^{\prime}\right)$ to $\mathscr{I}\left(\mathscr{I}\left(K^{N+1} ; \phi_{1}{ }^{\prime}\right), \hat{\phi}_{2}{ }^{\prime}, \cdots, \hat{\phi}_{q}{ }^{\prime}\right)$, to $\mathscr{I}\left(K^{N+2} ; \bar{\phi}_{2}{ }^{\prime}, \cdots, \bar{\phi}_{q}{ }^{\prime}\right)$ to $K^{N+q+1}$. Clearly $\Pi \circ \theta=\Psi_{3}^{\circ} \Psi_{2} \circ \Psi_{1}^{\circ} \Pi^{\prime}$. A calculation shows that $\bar{\phi}_{i}{ }^{\prime}=\left(\alpha_{i}\right) \in S^{\circ}(0, N+1)$, then the induction hypothesis applies to $\Psi_{3}$, and finally another calculation shows that $\Psi_{3} \circ \Psi_{2} \circ \Psi_{1}=\Psi$.

THEOREM 3.7. Every twist free minimal distal codimension one flow with non-trivial isotropy is a perturbation of an equicontinuous minimal co-dimension one flow with non-trivial isotropy.

Proof. By Theorem 2.3 it suffices to consider $\left(K^{p}, \mathscr{I}\left(K^{p+1}, Z_{f}^{q}\right), R^{q}\right)$. Let $\phi_{i}=\left(\alpha_{i}, f_{i 1}, \cdots, f_{i p}\right)=f\left(\cdot, e_{i}\right)$ and note $\alpha_{i}=\theta\left(e_{i}\right)$. Now Lemma 
3.7 applies and we can define $\left(K^{n}, R *^{q}\right)$ by $\left(z_{0}, \cdots, z_{n-1}\right)^{*} v=$ $\Theta\left(\Theta^{-1}\left(z_{0}, \cdots, z_{n-1}\right) v\right)$. Then $\left(K^{p}, K^{n}, R *^{q}\right)$ is a bitransformation group and Proposition 1.2 applies to it and $\left(K^{p}, K^{n}, R^{q}\right)$ because $\Pi \circ \theta=\Psi \circ \Pi^{\prime}$.

Corollary 3.8. If $\left(X, R^{n-1}\right)$ is a twist free minimal distal codimension one flow with non-trivial isotropy of rank $p$, then it can be assumed that $X=K^{n}$ and the orbits are cosets of a dense $(n-1)$-dimensional connected subgroup of $K^{n}$ containing a p-dimensional torus.

4. The Twisted Case. Let $\left(X, R^{n-1}\right)$ be a twisted minimal distal codimension one flow with non-trivial isotropy. By Corollary 2.5 the rank of its isotropy is $n-2$ and hence it has a cocycle representation of the form $\left(K^{n-2}, \mathscr{I}\left(K^{n-1} ; \phi\right), R\right)$ where $\phi=\left(\alpha, f_{1}, \cdots, f_{n-2}\right)$ and $\operatorname{deg} f_{i} \neq 0$ for some $i$. Let $m_{i}=\operatorname{deg} f_{i}, g_{i}(z)=z^{m_{i}}$, and $\psi=\left(\alpha, g_{1}, \cdots, g_{n-2}\right)$. As in the previous section we identify $\left.\mathscr{\mathscr { G }}\left(K^{n-1} ; \phi\right) / K^{n-2}, R\right)$ with $\mathscr{\mathscr { I }}(K ; \alpha)$, R).

Proposition 4.1. There exists a cocycle $\sigma$ of $(\mathscr{I}(K, \alpha), R)$ into $K^{n-2}$ such that $\left(K^{n-2}, \mathscr{I}\left(K^{n-1} ; \phi\right), R\right)$ is isomorphic to $\left(K^{n-2}, \mathscr{I}\left(K^{n-1} ; \psi\right), R_{\sigma}\right)$.

Proof. Let $h_{i}: K \times[0,1] \rightarrow K$ be a homotopy with $h_{i}(z, 0)=g_{i}(z)$ and $h_{i}(z, 1)=f_{i}(z)$. This provides a natural isotropy between $\psi$ and $\phi$, and using it we define a homeomorphism $\theta$ of $\mathscr{I}\left(K^{n-1} ; \phi\right)$ onto $\mathscr{I}\left(k^{n-1} ; \psi\right)$ by

$$
\begin{gathered}
\Theta\left(\left\langle\left(z_{0}, \cdots, z_{n-2}\right), t\right\rangle_{\phi}\right) \\
=\left\langle\left(\alpha \cdot z_{0}, h_{1}\left(z_{0}, t\right) \cdot z_{1}, \cdots, h_{n-2}\left(z_{0}, t\right) \cdot z_{n-2}\right), t\right\rangle_{\psi}
\end{gathered}
$$

for $t \in[0,1]$. Clearly $\Theta$ is equivariant with respect to the $K^{n-2}$ actions. Now $\theta$ induces the automorphism of $(\mathscr{I}(K ; \alpha), R)$ defined by $\langle z, t\rangle \rightarrow\langle\alpha \cdot z, t\rangle$, and we can complete the proof by applying Proposition 1.2 as we did in the proof of Theorem 3.5.

Consequently to obtain a result analogous to Theorem 3.5 in the twisted case we need a canonical representation of $\left(K^{n-2}, \mathscr{I}\left(K^{n-1} ; \psi\right)\right.$, $R)$. To do this we shift to the $\left(\mathscr{I}\left(K^{n-1} ; \psi\right), R^{n-1}\right)$ viewpoint and describe its lift to $R^{n}$.

Defining $p: R^{n} \rightarrow \mathscr{I}\left(K^{n-1} ; \psi\right)$ by $p(v)=\left\langle\exp v_{0}, \cdots, \exp v_{n-2}, t\right\rangle_{\psi}$ we obtain the universal covering of $\mathscr{I}\left(K^{n-1} ; \psi\right)$. The group $\Gamma$ of covering transformation is generated by $L_{j}(v)=v+e_{j}, 0 \leqq j \leqq n-2$ and $\tilde{\psi}(v)=\left(v_{0}+a, v_{1}+m_{1} v_{0}, \cdots, \quad v_{n-2}+m_{n-2} x_{0}, x_{n-1}-1\right)$ where $\exp a=\alpha$. Defining $\left(R^{n}, R^{n-1}\right)$ by $(x, v) \rightarrow\left(x_{0}, x_{1}+v_{1}, \cdots\right.$, $\left.x_{n-1}+v_{n-1}\right)$ we get a lift of $\left(\mathscr{I}\left(K^{n-1} ; \psi\right), R^{n-1}\right)$. 
Let $N$ denote the nilpotent Lie group consisting of all matrices of the form

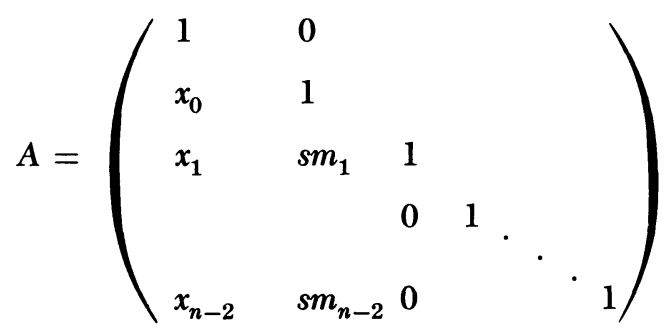

where $x_{i} \in R$ for $i=0, \cdots, x-2$ and $s \in R$. The map $h: R^{n} \rightarrow N$ given by

$$
h(v)=\left(\begin{array}{llll}
1 & 0 & \\
v_{0} & 1 & & \\
v_{1} & \left(1-v_{n-1}\right) m_{1} & 1 & \\
& & 0 & 1
\end{array}\right)
$$

is a homeomorphism of $R^{n}$ onto $N$. Let $\gamma_{j}$ denote the element of $N$ such that $s=0$ and $x_{i}=\delta_{i j}$ for $j=0, \cdots, n-2$ and let $\rho_{a}$ denote the element of $N$ such that $x_{i}=a \delta_{i 1}$ and $s=1$. It is easy to check that $h \circ L_{j} \circ h^{-1}$ is left multiplication by $\gamma_{j}$ and $h \circ \tilde{\psi} \circ h^{-1}$ is left multiplication by $\rho_{a}$. If we define $\left(N, R^{n-1}\right)$ by

$$
(A, v) \rightarrow A\left(\begin{array}{lllll}
1 & 0 & & & \\
0 & 1 & & & \\
v_{1} & -v_{n-1} m_{1} & 1 & & \\
& & 0 & 1 & \\
v_{n-2} & -v_{n-1} m_{n-1} & 0 & & 1
\end{array}\right)
$$

then $h$ is an isomorphism of $\left(R^{n}, R^{n-1}\right)$ onto $\left(N, R^{n-1}\right)$. The next step is to get rid of the $a$ in the above description of $\Gamma$. Let $\Gamma_{o}$ be the subgroup of $N$ such that $x_{i} \in Z$ for $i=0, \cdots, n-2$, and $s \in Z$. Note that $\Gamma_{o}$ is generated by $\left\{\gamma_{0}, \cdots, \gamma_{n-2}, \rho_{0}\right\}$. Let $\xi$ be the automorphism of $N$ defined by 


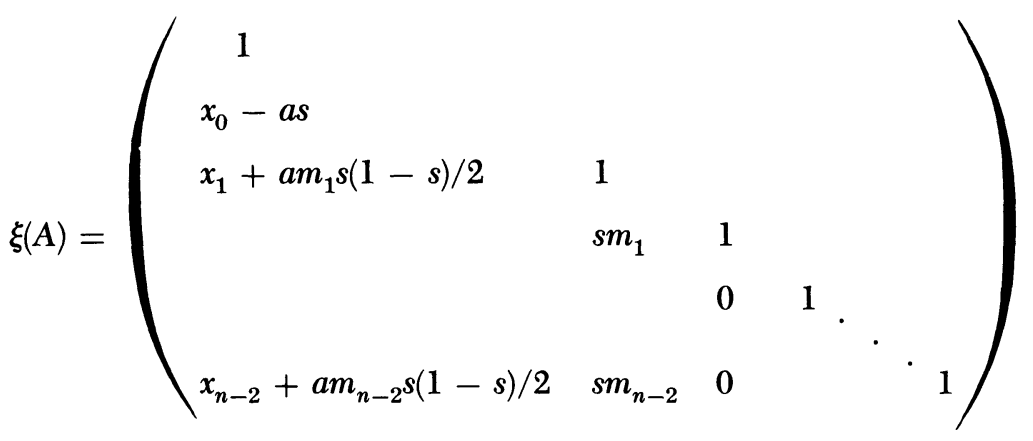

and check that $\xi\left(\gamma_{j}\right)=\gamma_{j}$ for $j=0, \cdots, n-2$ and $\xi\left(\rho_{a}\right)=\rho_{0}$. Therefore, $\left(\Gamma, R^{n}\right)$ is isomorphic to $\left(\Gamma_{o}, N\right)$ and $\mathscr{I}\left(K^{n-1} ; \psi\right)$ is homeomorphic to $\Gamma_{o} \backslash N=\left\{\Gamma_{0} A: A \in N\right\}$. The Lie algebra $\mathscr{L}(N)$ of $N$ consists of the matrices of the form

$$
\left(\begin{array}{llll}
0 & 0 & 0 & 0 \\
\beta_{0} & 0 & & \\
& \beta_{n} m_{1} & & \\
\beta_{n-2} & \beta_{n} m_{n-2} & 0 & 0
\end{array}\right) .
$$

Let $X_{j}$ denote the element of $\mathscr{f}(N)$ given by $\beta_{i}=\delta_{i j}$. Then the conjugate of $\left(N, R^{n-1}\right)$ under $\xi$ is given by commuting vector fields

$$
\left\{X_{1}, \cdots, X_{n-1},-a X_{o}+\frac{1}{2} a \sum_{i=1}^{n-2} m_{i} X_{i}+X_{n-1}\right\}
$$

and induces an action on $\Gamma_{o} \backslash N$ isomorphic to $\left(\mathscr{F}\left(K^{n-1} ; \psi\right), R^{n-1}\right)$. We will denote this flow by $\mathcal{N}(n, \bar{m}, a, T)$ where $n$ is a positive integer greater than $2, \bar{m}=\left(m_{1}, \cdots, m_{n-2}\right)$ is a non-zero element $Z^{n-2}, a$ is an irrational real, and $T$ is a linear transformation of $R^{n-1}$ onto $R^{n-1}$ which we use to change basis. The above with Proposition 4.1 proves the following:

TheOREM 4.2. Every twisted codimension one flow is a perturbation of some $=\mathcal{N}(n, \bar{m}, a, T)$.

\section{BiblogRAPHY}

1. D. De Riggi, The structure of codimension one flows, Dissertation, University of Maryland, 1976.

2. D. De Riggi and N. Markley, Shear distality and equicontinuity, Pacific Journal of Mathematics (to appear).

3. R. Ellis, Cocycles in topological dynamics, preprint. 
4. W. Gottschalk and G. Hedlund, Topological Dynamics, American Mathematical Society Colloquium Publications, Vol. 36, 1955.

5. N. Markley, Lifting dynamical properties, Mathematical Systems Theory 5 (1971), $299-305$.

Department of Mathematics, University of Maryland, College Park, MARYLAND 20742 